\title{
STUDY OF SOME FEATURES OF THE LASER OPTICAL-ACOUSTIC METHOD FOR TECHNICAL DIAGNOSTICS OF HEAT AND POWER ENGINEERING OBJECTS
}

\author{
Sharifov D.M. ${ }^{1}$, Sakipov K.E. ${ }^{1}$, Merzadinova G.T. ${ }^{1}$, Kaliyeva Zh.E. ${ }^{1,2}$, Userbaev M.T. ${ }^{3}$ \\ 1'L.N. Gumilyov Eurasian National University, 010008, Nur - Sultan,Kazakhstan \\ 2Institute of Scientific, Technical and Economic Research, 010008, Nur - Sultan,Kazakhstan \\ 3S.Seifullin Kazakh AgroTechnical University, 010011, Nur - Sultan,Kazakhstan
}

The article presents the diagnosis of heat power facilities. The distinguishing features of the applied method are shown in solving a number of technologically important diagnostic problems, in particular: quality control of pipe welds, determination of residual stress, structural, mechanical, elastic properties of materials. The physical foundations of the laser optoacoustic method for exciting ultrasonic waves are theoretically investigated. It is shown that this method is based on laser thermooptical excitation of broadband ultrasonic signals in a test object. Absorbed in a thin surface layer of the object under study, the laser pulse excites a broadband ultrasonic pulse. Propagating in an object, an excited ultrasonic pulse (either a transmitted or scattered ultrasonic signal with a high temporal resolution is recorded) carries information about the object under study.

Keywords: Laser optical-acoustic method, heat-power objects, defects.

\section{Introduction}

By definition, heat and power facilities are a complex of interconnected technological equipment (machines, installations, mechanisms, automation and control systems, engineering networks, etc.) intended for generating and transporting heat energy to the consumer. Characteristic features of heat-power objects are that they are under constant external influences (temperature, pressure and other physical loads) throughout almost their entire life cycle. Therefore, the safety and reliability of their operation largely depends on timely and high-quality technical diagnostics. As a rule, the structural foundations of many of these objects are made up of metal materials (screen pipes of boiler plants, discharge plants, steam generators, pipes and shut-off valves, etc.), which are especially exposed to high temperature and pressure differences.

It is known that the formation of defects and destruction of metal parts almost always begins with the formation of microcracks on the surface or deep under the surface of the material. The presence of a concomitant tensile stress on the surface of the studied objects leads to the growth of cracks and thus their further destruction. It is known that the formation of defects and destruction of metal parts almost always begins with the formation of microcracks on the surface or deep under the surface of the material. The presence of a concomitant tensile stress on the surface of the studied objects leads to the growth of cracks and thus their further destruction. Compressive stresses, though, prevent the growth of cracks, but contribute to their formation. If, in addition to the stresses arising from workloads, there are also so-called residual stresses in the metal that arose as a result of special processing or previous operation and remain in the absence of workloads, these stresses are summed up. Therefore, to study the suitability and increase the service life of metal products, it is very important to determine the sign and value of residual stresses in the product [1]. From this point of view, the use of more effective methods of technical diagnostics (TD) and non-destructive testing (NDT) of heat and power facilities plays a key role in the implementation and solution of tasks for the safety and reliability of their operation. 
As shown by the research on the technical condition of heat power facilities (for the main sources of heat - boiler equipment, heat networks, heat points, etc.) [1-16]. In the context of the regions of the Republic of Kazakhstan, currently more than half of them have long worked out their operating resources and need major repairs or complete replacement. In this regard, it becomes necessary to solve the problem of creating systems for monitoring, diagnostics and monitoring of these objects in order to prevent and reduce the risk of an accident. It is revealed that the frequency and nature of the level of accidents, damage to boilers (including Autonomous, low-power), heating networks, heat points and other auxiliary equipment, in the vast majority of the overall picture of the state of objects is similar and is primarily associated with:

- wear and tear of equipment due to the expiration of its service life (up to 65-70\%);

- leaks (micro-cracks) in the boiler sections and heat exchanger tubes;

- accumulation of scale and sludge in boilers and furnaces;

- formation of scale and leaks on the front and screen pipes of the boiler;

- a violation of the rules of operation and safety;

- exceeding the permissible values of the coolant pressure;

- non-compliance with modern conditions or poor-quality standards and operating rules (both for metal and polymer bases) of heat pipelines;

- lack of specialized diagnostic laboratories and automated systems of regular monitoring for early diagnostics and assessment of the technical condition of these facilities.

\section{A brief comparative analysis of the available methods of technical diagnostics and non-destructive testing.}

Currently, the following main methods of technical diagnostics and non-destructive testing of various technological objects are identified [11]: Visual and measuring; Ultrasonic; Acoustic emission; Radiation; Magnetic, Eddy current, Penetrating substances (capillary and leak detection), Vibrodiagnostic, Electric,Thermal, Optical and spectroscopy.

As a rule, each of these methods has its advantages and disadvantages, depending on the specific purpose and task of diagnostics. Also, the physical properties of materials and characteristics of research objects are of crucial importance when choosing a specific of NDT methods. Of course, when several methods are used to diagnose objects simultaneously, the probability of determining their real state increases. Depending on the spatial location of possible defects, they can be divided into surface, subsurface with a depth of 0.5 to $1.0 \mathrm{~mm}$ and internal with a depth of more than $1.0 \mathrm{~mm}$. Almost all methods are applicable for detecting surface defects, but as a rule, the most effective are visual-optical, magnetic powder and capillary, and for detecting subsurface defects - radiation, ultrasonic, eddy current, and magnetic powder. As practice shows, in the field of heat power engineering, optical, thermal and acoustic (ultrasonic) methods have received the greatest distribution (more than half in relation to other methods of non-destructive testing) [15].

Standard methods and diagnostic technologies based on the above methods are used for thermal power facilities (when examining the quality of welds, seams, joints, and the structural composition of the material). For example, for welded joints and metal structures, this technique is called styloscoping - the simplest type of qualitative spectral analysis for the presence of alloying elements in various metals and alloys. Such a diagnostic procedure is mandatory (according to State Standard 7122-81) for all heating metal surfaces (boiler installations, pipelines, etc.) and welded joints. This method allows you to clearly determine the presence of a certain composition of alloying elements in metal and alloys. In this way, you can control the quality and brand of the welding structure used. The device for stylescope diagnosis and study of spectra is called stylescope. Also, based on the method of capillary flaw detection, the method of color flaw detection (State Standard 18442-80), which is designed to detect surface and through defects, 
determine their location or extent (for extended defects of some types) and their orientation on the surface of the object under study. The essence of this method is the penetration of a liquid indicator into the defect, which is well wetting the test material, on the visual study of the material surface and on the subsequent registration of indicator traces [16].

The laser optical-acoustic ultrasonic method includes the features of these methods and is therefore the most versatile, informative, sensitive and in many other parameters (highresolution, visualization, imaging, velocities etc.) superior to many traditional methods of NDT. In particular, in contrast to the traditional ultrasonic method of diagnostics, the parameters (space-time) of the probing pulse allow creating and registering ultrasonic waves of wide frequency bands with the laser OA ultrasonic method. In the end, these characteristics allow you to get better images when visualizing defects and avoid the so-called "dead zones" in the objects under study. As noted, of particular interest is the development of combined methods based on laser optical-acoustic and laser-ultrasonic, which, mutually complementing each other, allow us to obtain more complete information about the object [6].

Laser optical-acoustic (photoacoustic) ultrasonic (LOAU) method - as a method of nondestructive testing is relatively "new" compared to other traditional methods. As its name implies, it appeared as a" laser" after the discovery of lasers and thanks to its unique properties, lasers brought the optical-acoustic method to a completely new level of development. Numerous scientific publications and books [2-10, 13-14], regularly international conferences on photoacoustic and photo-thermal phenomena (ICPPP), as well as the issue of the specialized international scientific journal "Photoacoustic", which has a high rating (Impact factor, 5.25 for 2018), indicate the prospects for the development and application of the LOAU method as one of the most popular methods of technical diagnostics In the previous works of the authors [9, 18], an analysis of the current state of this method was given and noted of great interest in the study of the fundamental thermo-physical properties (study of the thermal conductivity and thermal diffusivity) of various materials.

The purpose of this work is to evaluate the possibility of using the laser optical-acoustic ultrasonic method for technical diagnostics of various elements of heat-power objects.

\section{Theoretical foundation of the LOAU diagnostic method}

Laser opto-acoustic diagnostics is based on the photoacoustic) effect, the essence of which is the excitation of acoustic waves in the ultrasonic range $f=2 \times 10^{4} \div 10^{9}(\mathrm{~Hz})$ under the action of modulated or pulsed optical (often laser) radiation.Although the effect itself was discovered quite a long time ago (1880, by A. Bell), it received its second revival only after the discovery of lasers as sources of optical radiation with unique properties.It is the high intensity and correlation of spatiotemporal characteristics of lasers that allowed to excite acoustic waves of a fairly wide range, which together with the parallel level of development of signal processing technology contributed to the wide application and development of the OA method as a whole. Depending on the registration methods OA signals (in the frequency range), piezoelectric and microphone registration schemes are distinguished. As a rule, the piezoelectric OA method has a number of advantages: sensitivity (by several orders), frequency range, degree of visualization (imaging of the surface and subsurface defects), resolution, etc. Therefore, the piezoelectric OA method of registration is the most widely used in OA flaw detection. In the theoretical description of the OA diagnostic method, the thermooptic (thermoplastics) mechanism for generating optical-acoustic signals is mainly considered. It is assumed that a monochromatic laser pulse with a wavelength of $\lambda$ (or continuous with intensity modulation $f(t)=[1+\operatorname{mcos}(\omega t)])$, falls on the surface $(z=0)$ of the test sample (Fig.1.).

The test sample (blank) is considered opaque at the wavelength of laser radiation $\lambda$, i.e., it absorbs at least part of the energy of optical radiation.Further, in the zone of the laser pulse, due to the processes of thermodiffusion (change in the equilibrium state), on the surface and deep into the sample, the absorbed energy is converted into thermal energy, causing a local change in temperature 
and, consequently, the thermodynamic and elastic properties of the sample, subsequently generating acoustic waves (OA signal) at the frequency of the incident optical radiation $\mathrm{f}$. This process, as mentioned above, is called the thermooptic mechanism for generating acoustic waves.

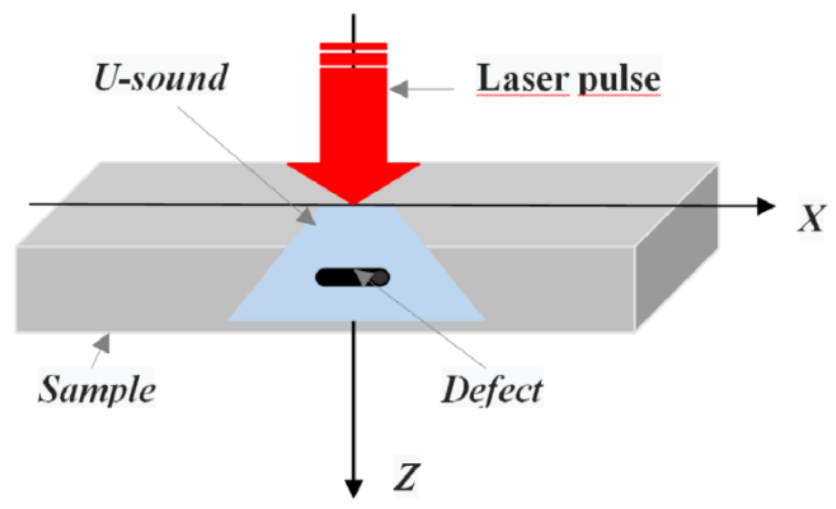

Fig. 1. Geometry of the problem

At the same time, all thermodynamic parameters of the test sample (object) are located far from the point of phase transitions. Theoretical equations describing the processes of thermooptic excitation of acoustic waves in homogeneous isotropic solid-state materials are well-studied and well-known $[2,8,13-14$, etc.]. They represent closed systems of wave equations for acoustic and temperature waves.Depending on the interaction of optical radiation (conditions of the input optical energy, parameters of the radiation source, optical and thermophysical properties of the object of study, etc.), the theoretical descriptions of the generation of OA signals may differ, although the physical essence of the effect remains. For example, for strongly or weakly absorbing optical radiation, the spatio-temporalheating regions and, accordingly, the processes of generating the OA signal change.

One of the important OA parameters is the length of thermal diffusion $\mu$, which allows for varying the frequency of probing laser pulses $\mathrm{f}$, to obtain and control the penetration depth of heat sources and the parameters of the excited ultrasonic radiation. It is related to the frequency of optical radiation modulation $\mathrm{f}$ and the thermophysical characteristics of the object of study as:

$$
\mu=\left(\frac{2 \chi}{\omega}\right)^{1 / 2}
$$

where, $\chi=\frac{k}{\rho \mathrm{C}_{p}}$ is the coefficient of thermal conductivity $\left(\mathrm{m}^{2} / \mathrm{sec}\right)$, $\mathrm{k}$ is the coefficient of thermal conductivity $(\mathrm{W} / \mathrm{m} \times \mathrm{K}), \rho$ is the density $\left(\mathrm{kg} / \mathrm{m}^{3}\right), \mathrm{C}_{\mathrm{p}}$ is the heat capacity $(\mathrm{J} / \mathrm{kg} \times \mathrm{K})$, and $\omega=2 \pi \mathrm{f}(\mathrm{Hz})$ is the cyclic frequency.

For the thermal and power facilities (mostly a metal materials) the value of the optical absorption coefficient is sufficiently $\operatorname{large}\left(\beta \approx 10^{6} \mathrm{sm}^{-1}\right)$ and accordingly, the optical absorption thickness (light penetration depth) $d \approx 1 / \beta$ is less.On the other hand, most metal materials are characterized by high values of the coefficient of thermal conductivity (thermal conductivity) and, accordingly, the length of thermal diffusion $\mu$ according to (1). This allows the conditions $d<<\mu$. to be met. As shown by the analysis of optical-acoustic parameters [2] for most solid-state materials, up to the frequencies of $10^{11} \mathrm{~Hz}$, the connection between heat and acoustic waves in the process of generating OA signals can be neglected, i.e. acoustic waves propagate adiabatically and thus heat waves do not have time to propagate over a distance of the order of the acoustic wave length during the oscillation period.These conditions allow us to solve the thermal and acoustic part of the problem separately. Therefore, to determine the temperature field $\mathrm{T}(\mathrm{z}=0, \mathrm{t})$, you can use the 
original equations of thermal conductivity with the corresponding boundary conditions (equality of temperature and heat flows at the boundaries, $\mathrm{z}=0$ ):

$$
\begin{aligned}
& \left\{\begin{array}{l}
\frac{\partial T_{1}}{\partial t}=\alpha_{1} \Delta T_{1} \\
\frac{\partial T_{2}}{\partial t}=\alpha_{2} \nabla T_{2}
\end{array}\right. \\
& \left.T_{2}\right|_{z=0}=\left.T_{1}\right|_{z=0}, \quad \rho_{2} c_{2} \alpha_{2} \partial T_{2} /\left.\partial z\right|_{z=0}=\rho_{1} c_{1} \alpha_{1} \partial T_{1} /\left.\partial z\right|_{z=0}+I_{0} f(t),
\end{aligned}
$$

where the indices denote, respectively, a transparent 1 and an absorbing 2 medium, $I(t, z=0)=I_{0} f(t)$ - intensity of laser radiation.

The equations for determining the generated acoustic field for the potential $\varphi$ (vibrational velocity $v$ ), are systems of wave equations (4) with the right part describing the distributions of thermal sources defined by (2) and (3):

$$
\left\{\begin{array}{l}
\frac{1}{\vartheta_{1}} \frac{\partial^{2} \varphi_{1}}{\partial t^{2}}-\Delta \varphi_{1}=-\beta_{1} \frac{\partial T_{1}}{\partial t} \\
\frac{1}{\vartheta_{2}} \frac{\partial^{2} \varphi_{2}}{\partial t^{2}}-\Delta \varphi_{2}=-\beta_{2} \frac{\partial T_{2}}{\partial t}
\end{array},\right.
$$

Here $p=\rho c v=\rho c(\operatorname{grad} \varphi), v=\operatorname{grad} \varphi$ and $\beta_{1}, \beta_{2}$ - are the effective thermal expansion coefficients of these media.

Boundary conditions describing the continuity of pressure and vibrational velocity at the interface of two media have the form:

$$
\left.\rho_{1} \varphi_{1}\right|_{z=0}=\left.\rho_{2} \varphi_{2}\right|_{z=0}, \quad \partial \varphi_{2} /\left.\partial z\right|_{z=0}=\partial \varphi_{2} /\left.\partial z\right|_{z=0}
$$

The systems of equations (2) and (4) with corresponding boundary conditions (3) and (5) represent a theoretical model of the processes of thermooptic excitation of OA signals and usually have their own methods and approaches for analytical solution (spectral, green functions, Fourier transform, Laplace, etc.) [2, 13-14]. The results of theoretical analysis show that the shape of the OA signal profile will repeat the envelope of the laser pulse (for a short duration of the laser pulse), i.e. it will not contain fluctuations that are characteristic of acoustic pulses excited by piezoelectric method. This is one of its characteristic features, which allows you to successfully use it when solving diagnostic problems. The parameters of the LOAU signal depend on a number of physically important properties of the objects under study (optical, thermal, elastic, geometric dimensions and acoustics). Therefore, the OA signal is quite informative and in certain cases, depending on the object of research, it is used in solving various problems of diagnostics (properties of gases with microphone detection, liquids and solids, porous, multilayer, biomedical, etc.).

\section{Application of the LOAU diagnostic method}

One of the main objects of heat energy production, as noted above, is a boiler plant (BP), which is under constant temperature and pressure changes. Therefore, at the same time, the BP is the most likely source of accidents and malfunctions in heat power facilities. The objects of technical control are: heating surfaces of boiler installations, smoke pumps, blow fan, auxiliary equipment, walling, fuel supply systems, economizers, regenerative air heaters, gas pipelines, automation, fuel treatment systems in solid fuel CU (mill, coal feeders and conveyors), fittings and other elements. Also, currently in the Kazakhstan, more than half of the heat and power equipment 
has developed an operational life, which is why the problem of creating control systems, technical diagnostics and monitoring of heat and power facilities is becoming more acute.

As a rule, low reliability of heating equipment leads to accidents and significant energy losses and, as a result, to high economic costs and a significant amount of repair and restoration work. It should be notedthat the improvement of technical diagnostics methods and the search for innovative methods are becoming more relevant. Thus, complementing the capabilities of other traditional methods of technical diagnostics, LOAU flaw detection is essentially innovative in this direction. The heat and power objects under investigation are mainly metal pipes of various diameters and thicknesses. Schematically, the principle of operation of LOAU flaw detectors for quality control of welds is shown in figure 2. After absorption of the laser pulse in the OA source, an ultrasonic pulse (longitudinal acoustic waves with a known amplitude and frequency spectrum) is excited in it, i.e., a probing (reference) pulse, which then propagates in the sample under study and is registered using a specially designed broadband piezoelectric receiver that is in acoustic contact with the sample.

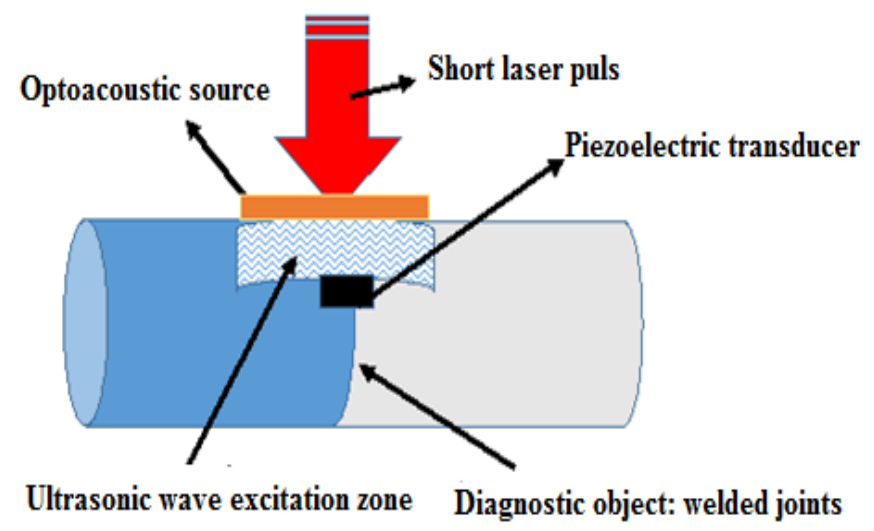

Fig.2. Principle of application of the LOAU flaw detector

Currently, many authors have developed and patented a number of experimental and semiindustrial flaw detectors based on laser OA excitation of broadband ultrasonic waves. For example, the ultrasonic laser multifunctional flaw detector UDL-2M is designed for ultrasonic examination of structural disorders of materials such as metals, composites, ceramics and plastics. As noted [17, 18], the generation of broadband acoustic pulses occurs due to the laser thermooptic mechanism of ultrasound excitation. The thickness of the test samples can be $0.1 \ldots 70 \mathrm{~mm}$, the cross dimensionsfrom $10 \mathrm{~mm}$.

\section{Conclusions}

The analysis of the possibility of using the LOAU diagnostic method for the examination of various heat and power facilities is carried out. It is noted that this method has a number of characteristic advantages in comparison with other traditional methods of ultrasound control, which makes it possible to successfully apply it. In particular, high resolution (tens of microns), allowing visualization of defects, the absence of a "dead zone" in the considered area of the object, also the high sensitivity to the change of sign of the impedance environment that allows to distinguish the defects of porous and dense inclusions, microcracks, etc. Thus, the analysis shows that the LOAU method, complementing the traditional methods of technical diagnostics, can be successfully applied in the technical diagnostics of various heat and power facilities.

\section{REFERENCES}

1 Ovchinnikov V.V.Weld defects and quality control of welds. Moscow, 2017, 224 p. [in Russian]

2 Gusev V.E., Karabutov A.A.Laser optoacoustics. American Institute of Physics, 1993, 203p. 
3 Tam A.C.Pulsed-laser generation of ultrashort acoustic pulses: Application for thin-film ultrasonic measurements. Appl. Phys. Lett. 1984, Vol. 45, No. 5, pp. 510-512.

4 Bychkov A., Simonova V., Zarubinet V., et al.The Progress in Photoacoustic and Laser Ultrasonic Tomographic Imaging for Biomedicine and Industry: A Review. Appl. Sci. 2018, Vol.8, 1931, pp. 1 - 26.

5 Wang L.V. Photoacoustic Imaging and Spectroscopy. Boca Raton: CRC Press. 2009, 499 p.

6 Bychkov A.S. Combined optical-acoustic and laser-ultrasonic tomography of media with inhomogeneities of acoustic properties and induced heat sources.Diss. PhD, Moscow. 2019, 128 p.

7 Zakrzewski J., Chigarev N., Tournat V., Gusev V. Combined Photoacoustic-Acoustic Techniquefor Crack Imaging. Int. J. Thermophys, 2010, Vol. 31(1), pp.199-207.

8 Sylvain Mezil. Nonlinear optoacoustics method for crack detection \& characterization. Diss. $\mathrm{PhD}$, Universite du Maine, Le Mans, France, 2012, 176 p.

9 Merzadinova G. T., Sakipov K. E., KalievaZ. E., et al. Laser optical-acoustic ultrasonic method of technical diagnostics of heat power facilities. Proceeding of the $11^{\text {th }}$ Intern. Scient.Conf. "Chaos and structures in nonlinear systems. Theory and experiment”, Karaganda, 2019, pp. 336-339.

10 PelivanovI.M., Kopylova D.S., Podymova N.B., Karabutov A.A.Optoacoustic method for determination of submicron metal coating properties:Theoretical consideration. J. Appl. Phys., 2009, Vol.106, pp. 0135071-0135078.

11 Ivanov E.A., Kotelnikov V.S., Shatalov A.A. et.al. Regulatory document. Rules for personnel certification in the field of non-destructive testing. Series 28. Issue 3. Team of authors. Moscow, 2010, $58 \mathrm{p}$.

12 State Standard: R53696-2009. National standard of the Russian Federation. Non-destructive testing. Optical methods. Terms and definitions. Moscow, Standardinform. 2010, 17 p.

13 Tam A. Applications of photoacoustic sensing techniques. Rev.Mod Phys. 1986, Vol. 58(2), pp.381.

14 Lyamshev L.M. Laser thermooptical excitation of sound. Moscow, 1989, 240 p.[in Russian]

15 Ivasev S.S., Girin A.V., Ravodina D.V. Methods of non-destructive testing. Krasnoyarsk, 2015, 112p. [in Russian]

16 KonstantinA. Flaw detection of welds: characteristics of the main types, rules of execution, advantages and disadvantages. Availabl at: https://elsvarkin.ru/texnologiya/kontrol/defektoskopiya-shvov.

17 Karabutov A.A., Kobeleva L.I., Podymova N.B., et al. Laser optical-acoustic method for local measurement of elastic modules of composite materials strengthened by particles. Technical acoustics. 2008, No. 19 , pp. 1-15.

18 Merzadinova G.T., Sakipov K.E., Sharifov D.M. et.al. Laser photoacoustics method for determination of the coefficients of thermal conductivity and thermal diffusivity of materials. Eurasian Physical Technical Journal, Volume 16, No. 1(31), 2019, pp. 94-98. 